\title{
ANÁLISE DOS CONTEÚDOS DE RORSCHACH DE PACIENTES COM DISTÚRBIO AFETIVO BIPOLAR*
}

\author{
CONTENT ANALYSIS OF RORSCHACH IN PATIENTS \\ WITH BIPOLAR AFFECTIVE DISORDER
}

Ana Valéria Guelli', André Jacquemin², Manoel Antônio dos Santos²

Psicóloga e bolsista ${ }^{1}$ da FAPESP; Docente ${ }^{2}$ do Departamento de Psicologia e Educação da Faculdade de Filosofia, Ciências e Letras da Universidade de São Paulo.

CorRESPONDÊNCIA: Ana Valéria Guelli. Rua Lafaiete, 1976, apto 9- Vila Seixas - CEP: 14015-080 - Ribeirão Preto - SP.

Telefone: (016) 635.33.52 - FAX: (016) 633.50.15 - FFCLRP - USP

GUELLI AV; JACQUEMIN A \& SANTOS MA dos. Análise dos contéudos de Rorschach de pacientes com distúrbio afetivo bipolar. Medicina, Ribeirão Preto, 29: 269-277, abr./set. 1996.

RESUMO: Foram analisados os conteúdos dos protocolos Rorschach de pacientes bipolares, utilizando o "Crivo de Representação de Si", a fim de avaliar a qualidade da representação de si e as modalidades de relação de objeto no grupo estudado.

Foi investigada uma amostra de 11 sujeitos, atendidos pelo Serviço de Psiquiatria do Hospital das Clínicas da Faculdade de Medicina de Ribeirão Preto - USP, com diagnóstico de transtorno afetivo bipolar, sendo 3 do sexo masculino e 8 do sexo feminino, com idades variando entre 22 a 67 anos e nível intelectual entre Médio e Médio Inferior.

Foram analisados os conteúdos, segundo as dimensões de integridade, vitalidade e o caráter realista, os tipos de ação, a diferenciação de gênero e os elementos qualitativos particulares, eventualmente introduzidos nas respostas.

A análise pluridimensional dos conteúdos evidenciou, no grupo estudado, indicadores de uma representação de si comprometida do ponto de vista da integridade e vitalidade, de uma fragilidade na estrutura da identidade pessoal e de modalidades relacionais pouco satisfatórias, sendo marcante a agressividade nas relações interpessoais.

UNITERMOS: Teste de Rorschach. Técnicas Projetivas. Distúrbio Bipolar. Auto-Imagem.

\section{INTRODUÇÃO}

A análise de conteúdos tem sido uma das preocupações freqüentes nos estudos com o teste de Rorschach. Segundo Aronow e Reznikoff ${ }^{1}$, esta área de estudo foi incrementada principalmente a partir da década de 40.

O estudo da "representação de si e de objeto", através do teste de Rorschach, inicia-se com Mayman" e Blatt ${ }^{3}$, que se preocupam em avaliar as dimensões cognitivas e afetivas do conteúdo de Rorschach.

Segundo Gaudriault e Baroth ${ }^{4}$, estes pesquisadores utilizam o teste de Rorschach para demonstrar um paralelismo entre o nível de organização do pensamento e o nível de desenvolvimento das relações de objeto. Centram-se no "conceito de objeto" e apontam que o sujeito internaliza imagens dos objetos, ou seja, "representações de objeto" e seus sentimentos

* Estudo financiado pela FAPESP 
frente a estas imagens. Imprimem, em sua história interpessoal, predisposições - para modalidades relacionais que estabelecerão a relação com os outros e com o mundo. Consideram que os conteúdos dos protocolos Rorschach compreendem as representações de objetos internalizados e revelam o repertório e a qualidade emocional destas representações, o grau de fusão em que se encontram entre si mesmos e os outros, a caracterização de si e dos outros, bem como, o tipo de expectativas que se estabelece frente às pessoas e às coisas do mundo, todos os aspectos da "relação de objeto" que potencializam o desenvolvimento social.

Lerner $^{5}$, utilizando a escala desenvolvida por Blatt para análise da representação de objeto, em um estudo com paciente depressivo, constata níveis baixos de diferenciação entre pensamento e ação e, sinais de uma representação de si, pouco estruturada e desvitalizada, notando-se mudanças no conteúdo e no mundo representacional do sujeito quando houve melhora da sintomatologia.

Milden et $\mathrm{al}^{6}$ estudam a utilidade do Rorschach para a compreensão dinâmica de pacientes psicóticos maníaco-depressivos, a partir de 100 protocolos, e concluem que pacientes que desenvolvem a psicose maníaco-depressiva apresentam uma vulnerabilidade quanto à representação de si. Segundo estes pesquisadores, o Rorschach mostrou-se um instrumento útil, que favorece a expressão, proporcionando informações a respeito do modo como os sujeitos representam-se e relacionam-se e, deste modo, contribuindo para a caracterização da psicose maníaco-depressiva e para o processo terapêutico.

Segundo Bolzinger ${ }^{7}$, contemporaneamente, uma especial contribuição no desenvolvimento da interpretação do teste de Rorschach, faz-se com o trabalho de Rausch de Traubenberg e Sanglade ${ }^{8}$, que introduz um instrumento de avaliação de representação de si e do objeto - o "Crivo de Representação de Si" - através da análise dinâmica dos conteúdos dos protocolos Rorschach, sob quatro aspectos: dimensão corporal, modalidades relacionais, identificação sexual e integração da identidade.

O conceito centralizador do trabalho é o de "representação de si" que carrega a influência do "conceito de objeto", desenvolvido por Mayman ${ }^{2}$ e Blatt ${ }^{3}$. Consiste numa noção inconsciente, que inclui a imagem corporal, enquanto experiência subjetiva, e também as relações que são por ela suscitadas e a estruturam. A representação de si traduz a forma de estar no mundo, associada ao sentimento de identidade pes- soal e às modalidades de relação que o indivíduo consegue estabelecer.

Segundo o trabalho de Rausch de Taubenberg e Sanglade ${ }^{8}$, o conceito referido abarca o conjunto de aspectos que são revelados pelo Rorschach. É proposto que a análise pluridimensional dos conteúdos se realize em torno de três eixos: o simbólico, apreendido pela categoria a que o conteúdo pertence; o formal, detectado pela diferenciação entre parte e totalidade e o eixo dinâmico, precisado pelo tipo de ação interpretado.

Considerando-se a relevância de uma abordagem dos fatores psicológicos característicos dos distúrbios psicóticos, o presente estudo pretende analisar a relação entre o desempenho no teste de Rorschach e o transtorno afetivo bipolar, através do "Crivo de Representação de Si", a fim de avaliar a qualidade da representação de si e as modalidades de representação de objeto no grupo em estudo. Pretende-se, também, verificar a presença ou não de características particulares nas categorias de análise do referido instrumento de interpretação para o grupo estudado.

É preciso frisar o caráter preliminar e exploratório do presente trabalho, em que tentamos delimitar um campo de estudos, criando a possibilidade de outras investigações mais aprofundadas.

\section{MÉTODO}

\section{Sujeitos}

Foram sujeitos deste estudo 11 pacientes psiquiátricos, com idades variando entre 22 e 67 anos, sendo 3 do sexo masculino e 8 do sexo feminino. No que se refere ao estado civil, a amostra compõe-se de 6 casados, 3 solteiros, 1 amasiado e 1 desquitado. Em relação ao exercício da profissão, o grupo distribui-se em 8 ativos profissionalmente, 2 inativos e 1 aposentado.

Todos os pacientes receberam diagnóstico clínico de transtorno afetivo bipolar, sendo 6 pacientes, atendidos pelo Ambulatório de Seguimento Medicamentoso do Hospital das Clínicas - Faculdade de Medicina Ribeirão Preto - USP, 4 pacientes, atendidos pela Enfermaria de Psiquiatria do referido hospital, em regime de internação, e apenas 1 paciente, atendido pelo Hospital Dia da faculdade citada, em regime de semi-internação.

A diferenciação dos sujeitos em termos de subtipos de transtorno afetivo bipolar é a seguinte: 6 pa- 
cientes apresentavam episódio maníaco, sendo 5 sem sintomas psicóticos e 1 com sintomas psicóticos, 4 apresentavam episódio hipomaníaco e 1 apresentava episódio misto.

O diagnóstico clínico foi estabelecido para cada paciente, de acordo com a nomenclatura e critérios diagnósticos da CID- $10^{9}$, por um médico psiquiatra com experiência na área.
O nível intelectual foi avaliado através do INV - Forma C. De acordo com as normas brasileiras, elaboradas por Weil e $\mathrm{Nick}^{10}$, a capacidade intelectual de 7 sujeitos foi classificada no nível Médio Inferior e, de 4 sujeitos, no nível Médio. Portanto, a amostra apresentava uma tendência à normalidade quanto ao nível intelectual, o que se constituiu em um critério de inclusão dos sujeitos para este estudo.

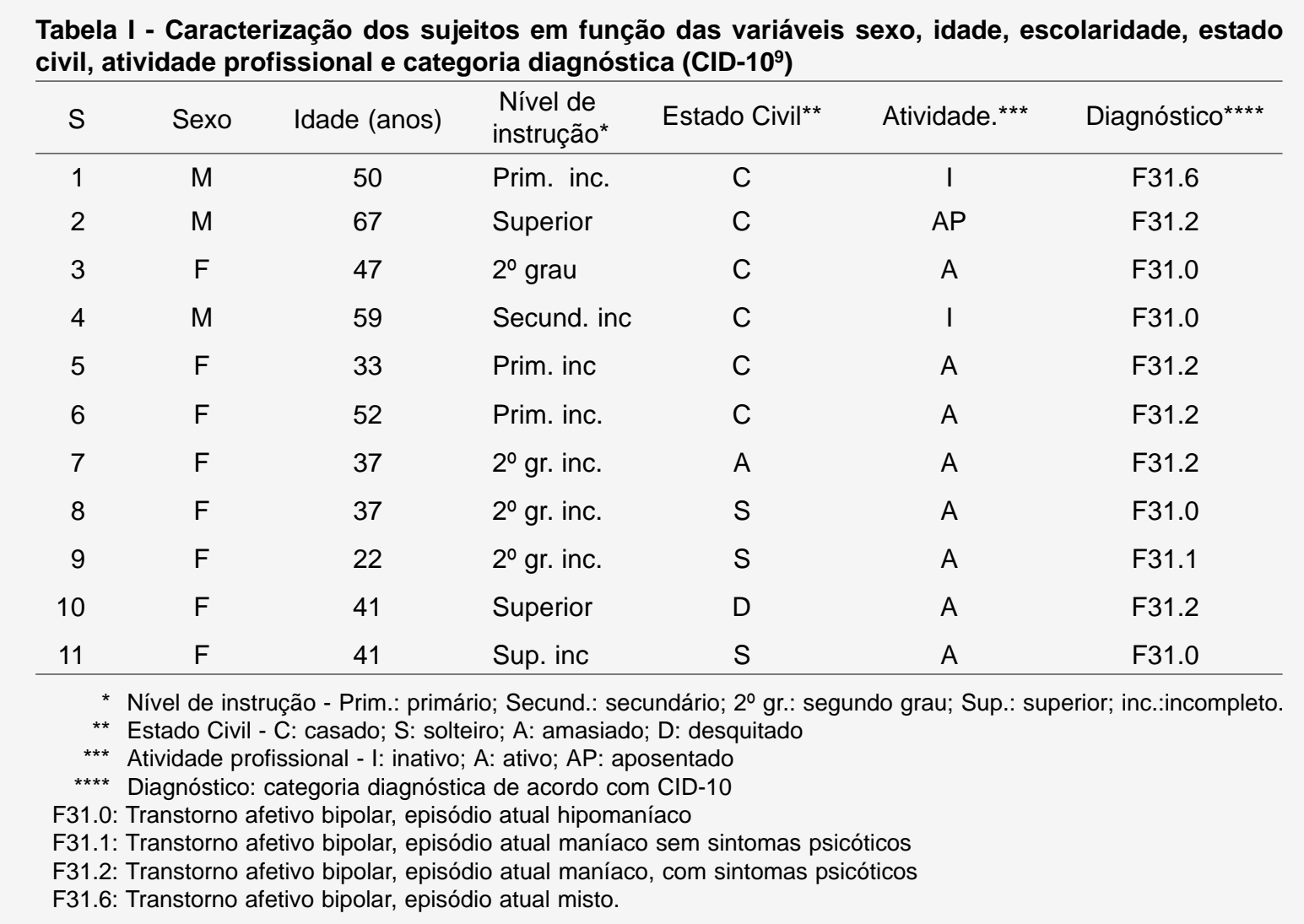

\section{PROCEDIMENTO}

Foi realizada primeiramente uma entrevista semi-estruturada, a fim de esclarecer os limites e objetivos da pesquisa, a duração e natureza das tarefas, de estabelecer o "rapport" com o sujeito e de obter dados de anamnese, significativos para o próposito deste trabalho. Este procedimento foi padronizado para todos os sujeitos.

A primeira sessão de testagem foi dedicada à aplicação do teste INV - Forma C. Uma vez que os resultados desta técnica não mostrassem evidência ou suspeita de deficiência mental ou "deterioro" mental, em função de quadro psicopatológico, o paciente era incluído na amostra.

Na segunda sessão, foi aplicado o teste de Rorschach. A aplicação e a apuração do teste de Rorschach foram conduzidas segundo as normas propostas pelo autor ${ }^{11}$, e demais reformulações, introduzidas por Anzieu e Chabert ${ }^{12}$ e Rausch de Traubenberg ${ }^{13}$.

\section{Tratamento dos Dados}

A cotação dos protocolos foi feita por dois juízes independentes. Quando ocorria discordância, um ter- 
ceiro avaliador era consultado e decidia-se de acordo com seu parecer. Foram observados os padrões normativos, estabelecidos para o meio brasileiro por Augras et al. ${ }^{14}$.

A análise de conteúdo foi realizada utilizando-se o "Crivo de Representação de Si" (Rausch de Traubenberg e Sanglade ${ }^{8}$ ). A cotação realizada, através deste instrumento, considerou o substantivo, o verbo e os adjetivos dados na resposta, de acordo com a categoria a qual pertence o conteúdo, a diferenciação entre o todo e as partes, o tipo de ação inscrito, diferenciação sexual dos personagens ou dos animais interpretados e os elementos qualitativos particulares introduzidos nas respostas. As freqüencias encontradas foram transformadas em porcentagem e comparadas com a literatura.

\section{RESULTADOS E DISCUSSÃO}

\section{1 - Imagem corporal}

A abordagem da dimensão corporal será realizada a partir dos resultados da primeira coluna do "Crivo", que exprime a imagem corporal da representação de si, segundo o caráter de vitalidade e integridade e o nível de organização.

A Tabela II corresponde à primeira coluna do "Crivo". Tal tabela apresenta a distribuição das respostas fornecidas pelos 11 sujeitos estudados, segundo as categorias de conteúdo que exprimem o objeto representado, conforme pertença ao mundo humano animal ou inanimado.

Nota-se que a categoria referente ao mundo animal $(56,0 \%)$ é predominante, quando comparada às categorias pertinentes ao mundo inanimado (28.3\%) e ao mundo humano $(15.7 \%)$. Tendo em vista as proporções médias esperadas para cada uma destas categorias (H\% entre $15 \%$ e $20 \%$ e A\% entre $35 \%$ e $50 \%$ do total de respostas, segundo Augras et $\mathrm{al}^{14}$ ), nota-se que a freqüência de conteúdo humano apresenta-se dentro do intervalo médio esperado, enquanto que a frequiência obtida na categoria de conteúdo animal aparece elevada, sinalizando uma tendência do grupo a recorrer a mecanismos de pensamento mais estereotipados e automáticos.

O mundo animal constitui-se em uma classe que parece favorecer a percepção de totalidade (44.6\%), sobre os detalhes $(10.8 \%)$, bem como a percepção realista $(52.1 \%)$ sobre a irrealista $(3.8 \%)$, o que coaduna com os resultados obtidos por Fazzani et $\mathrm{al}^{15}$ de um estudo sobre os dinamismos psíquicos de pacientes psicóticos maníaco-depressivos, através da análise de 15 protocolos Rorschach.

Com relação aos conteúdos pertencentes ao mundo inanimado, observa-se uma ênfase maior na categoria "botânica" (7,3\%), que parece ser indicativo de estereotipia, com o mesmo sentido observado no índice $\mathrm{A} \%$.

No que se refere aos conteúdos pertencentes ao mundo humano, apenas $6.0 \%$ das respostas corresponde a percepções realistas e de totalidade. Estes dados parecem indicar uma dificuldade de perceber a imagem humana de forma mais integrada e realista, visto o modo como o mundo humano aparece constituído pelos conteúdos anatômicos (3.3\%), pelos conteúdos parciais (4.9\%) e pelas percepções irrealistas $(1.1 \%)$. Segundo Lerner ${ }^{5}$, a diferenciação da figura humana em baixo nível de desenvolvimento $(\mathrm{Hd},(\mathrm{H})$ e Anat) é indicativo de uma representação de si e dos outros pouco estruturada. Evidenciou-se, assim, a vivência de não integridade corporal e a tendência do grupo estudado a recorrer a mecanismos de fragmentação e desvitalização, frente à representação humana, caracterizando uma representação de si, pobremente estruturada e pouco vitalizada.

O mundo, tal qual aparece organizado na natureza, indo de níveis de organização mais elementares (seres inanimados) até os mais complexos (seres vivos), é interpretado de modo desigual pelo grupo aqui estudado. Observa-se maior facilidade na interpretação de seres vivos, situados em um nível de evolução médio (animais). Alguns aspectos qualitativos das elaborações referentes aos conteúdos animais são, positivamente, superiores aos observados nos conteúdos humanos. Na categoria referente aos conteúdos inanimados, observa-se a ênfase no mundo vivo (Botânica).

A representação humana é a mais comprometida do ponto de vista de dinamismo e vitalidade que os sujeitos infundem nos perceptos. Na amostra estudada, um número considerável destas respostas é interpretado em nível primário, e até mesmo arcaico, sem a utilização de mecanismos de controle que corrijam estas distorções. Tais mecanismos de controle aparecem mais ativos na interpretação de outros conteúdos do teste, permitindo a elaboração de respostas mais neutras e mais adaptadas, como nos conteúdos animais. Nota-se, assim, uma diferença no manejo de conteúdos animais e humanos. 
TABELA II - Distribuição percentual das respostas, em função das categorias de conteúdo correspondentes ao objeto representado, conforme pertença ao mundo humano, animal ou inanimado (1를 coluna do crivo de representação de $s i)(n=11)$.

\begin{tabular}{|c|c|c|c|}
\hline & Primeira Coluna & $\mathrm{F}$ & $\%$ \\
\hline \multicolumn{2}{|c|}{ Mundo Humano } & 29 & 15.7 \\
\hline $00:$ At & Anatomia & 6 & 3.3 \\
\hline 002 & para anatomia óssea & 2 & 1.1 \\
\hline 003 & para anatomia visceral & 3 & 1.6 \\
\hline 004 & para o sangue, veias, artérias & 1 & 0.5 \\
\hline $03: \mathrm{H}$ & Humano definido pela função & 7 & 3.8 \\
\hline $07: \mathrm{Hd}$ & Parte do corpo que não a cabeça & 6 & 3.3 \\
\hline $01: \mathrm{H}$ & Conteúdo humano inteiro & 4 & 2.2 \\
\hline $08: \mathrm{Hdc}$ & Cabeça humana ou parte da cabeça humana & 3 & 1.6 \\
\hline $06: \mathrm{HA}$ & Mistura dos reinos humano e animal & 2 & 1.1 \\
\hline $02:$ Hcri & Conteúdo humano criança & 1 & 0.5 \\
\hline \multicolumn{2}{|c|}{ Mundo Animal } & 103 & 56.0 \\
\hline $11: A$ & Animal inteiro & 75 & 42.4 \\
\hline $17:$ Ad & Outros que não boca, dentes, chifres, pinças & 14 & 7.6 \\
\hline $14:(A)$ & Animais irreais e revestidos de caráter de onipotência & 4 & 2.2 \\
\hline $19:(\mathrm{Ad})$ & & 3 & 1.6 \\
\hline $18: \mathrm{Ad}$ & Boca, dentes, chifres e pinças & 3 & 1.6 \\
\hline $100:$ & Anatomia animal & 1 & 0.5 \\
\hline 103: & para anatomia visceral & 1 & 0.5 \\
\hline \multicolumn{2}{|c|}{ Mundo Inanimado } & 52 & 28.3 \\
\hline 30 : & Botânica & 15 & 8.2 \\
\hline 20 : & Objetos quaisquer & 7 & 3,8 \\
\hline $26:$ & Objetos ocos & 5 & 2.7 \\
\hline $29:$ & Elementos da Natureza & 4 & 2.2 \\
\hline 52 : & Materiais & 3 & 1,6 \\
\hline $25:$ & Arquitetura & 3 & 1.6 \\
\hline $31:$ & Geografia & 3 & 1.6 \\
\hline 21: & Detalhes de objeto & 2 & 1.1 \\
\hline $23:$ & Vestimentas e acessórios & 2 & 1.1 \\
\hline $28:$ & Objeto a motor & 2 & 1.1 \\
\hline $40:$ & Alimentação & 2 & 1.1 \\
\hline 61: & Símbolos & 2 & 1.1 \\
\hline $32:$ & Biologia & 1 & 0.5 \\
\hline 60 : & Abstrações & 1 & 0.5 \\
\hline \multicolumn{2}{|l|}{ Total } & 184 & 100.0 \\
\hline
\end{tabular}




\section{2 - Modalidades de relação}

O modo de relação será apreendido através da segunda coluna do "Crivo", que especifica o tipo de relação investida e sua intensidade, e assim, corresponde ao modo de entrar em contato com o objeto.

A Tabela III mostra a distribuição percentual das respostas, categorizadas em termos do tipo de ação e do tipo de interação. Tal tabela corresponde à segunda coluna do "Crivo".

Observa-se que, entre todas as categorias da segunda coluna, mostra-se predominante aquela referente à simples denominação estática (61.4\%). Nota-se a tendência no grupo estudado para neutralizar a reciprocidade, denotando dificuldades na relação destes indivíduos com o mundo, marcada por uma tendência a contatos despojados de dinamismo e reciprocidade. Segundo Tognazzo e Lagrasta ${ }^{16}$, a ausência de interação sugere resolução insatisfatória da fase simbiótica, indicativo de dificuldade de diferenciação e de uma representação de si, pobremente estruturada.

A análise do manejo da agressividade aponta, conforme Tabela III, que o modo pelo qual a agressividade se expressa é predominantemente implícito (3.8\%) (categoria 80 ) e em menor frequiência (1.1\%) de forma franca, explícita (categoria 8). Uma proporção, também, pequena $(1.1 \%)$ das respostas exprime agressividade sofrida (categoria 10).

Estes dados apontam uma tendência do grupo a reprimir a expressão clara da agressividade, ainda que se observe, quando se tem em vista o total de posições agressivas (8.2\%), a manifestação intensa de impulsos agressivos. Yazigi et al. ${ }^{17}$, pesquisando pacientes com desordem bipolar, confirmam estas características (agressividade e desconfiança nas relações interpessoais).

No grupo aqui estudado, aponta-se uma tendência a manifestar a agressividade implicitamente, o que aparece, também, subjacente à tendência para utilização da desvitalização, expressa pela categoria "desenho e caricatura" (1.1\%) e "estátua e múmia" (1.1\%), como defesa que favorece o distanciamento do contato com objetos que apresentam um caráter agressivo.

Observa-se, ainda, uma tendência à fragmentação, expressa pela categoria "imagem incompleta" (2.2\%) e pela percepção de conteúdos humanos parciais $(4.9 \%)$, já comentada na análise da primeira coluna. A vivência de fragmentação interna da representação corporal parece traduzir, de certa forma, o resultado da repressão dos impulsos agressivos, que não podendo se manifestar diretamente, extravazam sob a forma de um comprometimento do aparato perceptivo, comprometendo a integridade das representações psíquicas e distorcendo a natureza do objeto. A utilização da fragmentação e desvitalização nos sujeitos em estudo, como defesa contra o estabelecimento de relação, que se evidencia também nas respostas "imagem em espelho", como expressão do retraimento narcísico, parece ser insuficiente, por vezes, para conter os impulsivos agressivos, que se evidenciam nas respostas de agressividade dita franca, bem como nas respostas "ser humano ou animal morto" $(2.2 \%)$.

Tabela III - Distribuição percentual das respostas, em função do tipo de ação e de interação, projetados nos perceptos. ( $2^{a}$ coluna do crivo de representação de si) $(n=11)$

\begin{tabular}{rlrr}
\hline \multicolumn{1}{c}{ Segunda Coluna } & $\mathbf{F}$ & $\%$ \\
\hline 12: & Denominação simples ou postura não implicando K ou kan & 113 & 61.4 \\
7: & Ação simples e postura implicando K ou kan & 22 & 12.0 \\
3: & Ação bilateral de caráter neutro & 16 & 8.7 \\
80: & Caráter agressivo ou ameaçador sem ação & 7 & 3.8 \\
1: & Interação recíproca positiva & 7 & 3.8 \\
15: & Ser humano ou animal morto & 4 & 2.2 \\
$16:$ & Imagem incompleta & 4 & 2.2 \\
$11:$ & Imagem em espelho & 3 & 1.6 \\
8: & Ação com caráter agressivo & 2 & 1.1 \\
13: & Desenho, caricatura & 2 & 1.1 \\
14: & Estátua, múmia & 2 & 1.1 \\
10: & Ação sofrida & 2 & 1.1 \\
\hline Total & & $\mathbf{1 8 4}$ & $\mathbf{1 0 0 . 0}$ \\
\hline
\end{tabular}




\section{Identidade sexual}

A abordagem desta dimensão far-se-á através da terceira coluna do "Crivo", que precisa a identificação sexual, de acordo com seu nível de determinação e estabilidade.

A Tabela IV, que corresponde à terceira coluna do "Crivo", mostra a distribuição das respostas conforme o sexo dos personagens e animais interpretados.

A definição do sexo das figuras foi utilizada como indicador do nível de estruturação de identidade sexual, conforme propõem Rausch de Traubenberg e Sanglade $^{8}$. Observou-se definição sexual no sentido do próprio sexo dos sujeitos em apenas $37.4 \%$ das respostas, enquanto $40.6 \%$ das respostas apontam definição sexual no sentido oposto, em $16.3 \%$ o sexo não é precisado e em 5,7\% aparecem "ambivalentes, bissexuais ou instáveis". Segundo Osório ${ }^{18}$, a identidade sexual corresponde ao sentimento de pertencer à determinada categoria sexual, acompanhado da renúncia ao sexo oposto. A predominância das percepções do sexo ao qual não pertence o indivíduo, acrescida dos índices que expressam ambivalência e indeterminação são sugestivos do primitivismo, com que é elaborada a definição e a identidade sexual, e de uma representação de si pouco integrada.

Tabela IV - Distribuição percentual das respostas, de acordo com as categorias referentes à identificação sexual, projetadas nos perceptos ( $3^{\mathrm{a}}$ coluna do crivo de representação de $\left.\mathbf{s i}\right)(\mathrm{n}=11)$

\begin{tabular}{lrr}
\hline Terceira Coluna & $f$ & $\%$ \\
\hline gênero oposto & 50 & 40.6 \\
mesmo gênero & 46 & 37.4 \\
não precisado & 20 & 16.3 \\
ambivalente, instável & 7 & 5.7 \\
\hline Total & $\mathbf{1 2 3}$ & $\mathbf{1 0 0 . 0}$ \\
\hline
\end{tabular}

\section{Diferenciação e integração da identidade pessoal}

Estes aspectos serão avaliados através da quarta coluna do "Crivo", que refere-se aos elementos qualitativos particulares, introduzidos nas respostas.

Entre todas as categorias da quarta coluna, ocorre com maior frequiência aquela referente a "conteúdos deteriorados" $(2.7 \%)$. Estas respostas podem indicar uma dificuldade na integração da identidade destes sujeitos e, conseqüentemente, dificuldade na discriminação e separação do indivíduo em relação a suas imagens parentais paterna e materna $(2.2 \%$ e $1.6 \%$ respectivamente), processos fundamentais para a individuação, conforme aponta Osório ${ }^{18}$. Esta hipótese se reforça tendo em vista as frequiências das categorias "caráter unilateral"(2.2\%), "gêmeos, siameses" (1.1\%) e "sombras, marcas e traços" ( $0.5 \%)$, que apontam para uma dificuldade de relação e de diferenciação entre si e o outro, reafirmando os sinais de dificuldade de integração de identidade, no grupo em estudo.

\section{CONCLUSÃO}

A análise pluridimensional dos conteúdos permitiu evidenciar algumas tendências básicas no grupo estudado. O modo, segundo o qual os sujeitos se organizam e se situam nas interações que estabelecem com o mundo, estreitamente vinculadas com a organização de seu mundo objetal, apresentou-se sustentado sobre um sentimento de identidade pouco estruturado, é resultado de uma "representação de si" comprometida do ponto de vista da integridade e vitalidade, calcada em modalidades relacionais insatisfatórias.

A pouca estruturação do conceito corporal e a representação de si empobrecida, desarticulada e desvitalizada, parecem ser características de personalidade pobremente estruturada. Isto fica evidente quando são considerados os conteúdos humanos e anatômicos, que sinalizam a impossibilidade de integrar as diferentes experiências emocionais e sensoriais que, uma vez verificadas, permitiriam estruturar um saber sobre o próprio corpo. É como se o corpo fosse vi- 
venciado por estes pacientes, como uma entidade dispersa, sem uma fronteira delimitadora e sem uma solução de continuidade no tempo e no espaço. Isto pode estar de algum modo relacionado com a sensação de perda dos limites do ego, como no caso dos psicóticos maníaco-depressivos, os estados maníacos, com os delírios de grandiosidade e os sentimentos de onipotência que os caracterizam, conforme descrito por Kaplan ${ }^{19}$ e Fenichel ${ }^{20}$.

As dificuldades de unificação da representação corporal implicam, também, uma impossibilidade de estabelecer e representar relações objetais duradouras e estáveis. O grupo revelou um esforço para superar uma dicotomia entre a imagem humana desvitalizada e fragmentada, que o impede de identificar e estabelecer contatos interpessoais mais adequados, e sua organização defensiva mais primitiva, caracterizada por mecanismos de desvitalização, onipotência e negação.

Os perceptos tendem a servir à canalização da agressividade, evidenciando insuficiência de mecanismos de sublimação. Observa-se que o grupo, ainda, recorre a outras defesas, como a estereotipia e a contenção da capacidade imaginativa que, no entanto, mostraram-se ineficazes para controlar as manifestações impulsivas. A agressividade é manejada de um modo primitivo, o que torna o contato com o outro ameaçador, caracterizando dificuldade frente aos relacionamentos, falta de confiança nos contatos e imaturidade afetiva, que dificultam a ação do sujeito no meio.

Um baixo nível de elaboração da representação corporal reflete-se no conceito de identidade sexual, uma vez que afeta as relações objetais. Os índices obtidos sugerem um primitivismo da elaboração da diferenciação e da identidade sexual, freqüentemente patenteado na indiferenciação dos perceptos, na dificuldade de conferir uma maior especificidade aos conteúdos, como por exemplo a atribuição da ação, papel sexual adequado, etc. Estes dados parecem sugerir dificuldades no desenvolvimento da identidade sexual, o que resulta em prejuízo no desenvolvimento emocional, produzindo profunda imaturidade afetiva.

Portanto, na base do perfil de personalidade apresentado pelos pacientes psicóticos estudados, percebemos uma influência da capacidade de identificação relacionada, provavelmente, à insegurança, à inadequação ou ausência das figuras de identificação durante a infância, o que condiciona perturbações no desenvolvimento do eu e dificuldades na relação com o outro e com o mundo, em um sentido amplo. Nessa medida, parece essencial precisar quais atitudes são fundamentais desses indivíduos face a si mesmos e aos outros. Essa avaliação mostrou-se útil, no caso dos sujeitos estudados, notadamente quando vem a complementar a análise proporcionada pela avaliação clássica dos índices, tradicionalmente, investigados no teste de Rorschach.

\section{AGRADECIMENTO}

Agradecimento Especial a Dr. Jaime Eduardo Cecílio Hallak, Médico Assistente da Enfermaria de Psiquiatria do Hospital das Clínicas da F.M.R.P.-U.S.P. Departamento de Neurologia e Psicologia Médica, pela assessoria na área de diagnóstico clínico.

GUELLI AV; JACQUEMIN A \& SANTOS MA dos. Content analysis of Rorschach in patients with bipolar affective disorder. Medicina, Ribeirão Preto, 29:269-277, apr./sept. 1996.

ABSTRACT: The present study investigated the contents of Rorschach protocol in bipolar patients with were analyzed with the use of the "Self-Representation Sieve". The purpose was to analyze the quality of self-representation and the several types of the object-relationships in the studied group.

The sample was the eleven patients attended by the Psychiatry Service at the Hospital das Clínicas in the Faculdade de Medicina de Ribeirão Preto - USP. The patients had bipolar affective disorder diagnostic. The characteristics the sample were: three men and eigth women, with Medium and Inferior Medium intelectual level, with age groups ranging between twenty-two and sixty-seven years .

The Rorschach contents were analyzed considering their integrity and vitality dimensions, realistic character, types of action, sexual differentiation, and the qualitative elements casually introduced in the answers.

The contents multidimensional analysis showed, in the investigated group, signs of damaged self-representation, fragility in the personal identity structure and the relational modalities less satisfactories. A marking agressiveness in the interpersonal relations was observed.

UNITERMS: Rorschach Test. Projective Techinics. Bipolar Disorder. Self-Concept. 


\section{REFERÊNCIAS BIBLIOGRÁFICAS}

1 - ARONOW E \& REZNIKOFF M. Rorschach content interpretation. Grune \& Stratton, New York, 1976.

2 - MAYMAN M. Object-representations and objectrelationships in Rorschahch responses. J. Proj Techn \& Pers. Assess 31: 17-24, 1967.

3 - BLATT SJ. Leves of object representation in anaclit and introjective depression. Psychoanal Study Child 29: 107-157, 1974.

4 - GRAUDRIAULT P \& BARITH J. La Représentation d'Objet dans le Rorschach. XIII Congrès International du Rorschach et des Méthodes Projectives. Paris, 1990 (Mimeografado).

5 - LERNER H. An object representation approach to psychostructural change: A clinical ilustration. J Pers Assess 47: 314-323, 1983.

6 - MILDEN RS LUDOLPH PS \& LERNER HD. The Rorschach and affective disorders: The role of projective testing in a descriptive psychiatric model. In:LERNER HD \& LERNER PM, eds. Primitive mental states and the Rorschach. International Universities Press, New York, p.425-439, 1988

7 - BOLZINGER A. La Pratique et les Praticiens du Rorschach. Bull Psychol 43 (14/17): 631-636, 1990.

8 - RAUSCH DE TRAUBENBERG N \& SANGLADE A. Représentation de Soi et Relation d'Objet au Rorschach Grille de Représentation de Soi - Analyse comparée des résultats d'adolescents malades psychiques et malades somatiques. Rev Psychol Appl 34: 41-57, 1984.

9 - ORGANIZAÇÃO MUNDIAL DA SAÚDE. Classificação dos transtornos mentais e de comportamento da CID-10: descrições clínicas e diretrizes diagnósticas. Artes Médicas Porto Alegre,1993.

10 - WEIL P \& NICK E. O Potencial de inteligência do brasileiro. CEPA, Rio de Janeiro 1971
11 - RORSCHACH H. Psicodiagnóstico. 3a ed. Mestre Jou, São Paulo 1978

12 - ANZIEU D \& CHABERT C. Les méthodes projectives. $8 a$ ed. Presses Universitaires de France, Paris, 1987.

13 - RAUSCH DE TRAUBENBERG N. La pratique du Rorschach. 6a ed. Presses Universitaires de France, Paris 1990.

14 - AUGRAS M. org. Teste de Rorschach: Atlas e Dicionário: padrões preliminares para o meio brasileiro. 6a ed. Editora da Fundação Getúlio Vargas, Rio de Janeiro 1986.

15 - FAZZANI NETO R COELHO L \& MENDES FILHO RB. Dynamismes Psychiques Observés à travers des Protocoles de Rorschach de Patients avec Psychose Endogène. XIII Congrès International du Rorschach et des Méthodes Projectives. Centre de Psychologie Appliquée, Paris: 1990. résumé 291.

16 - TOGNAZZO DP \& LAGRASTA D. Image de Soi et Aspects Relationnels au Rorschach dans l'Obésité et dans l'Anorexie. XIII Congrès International du Rorschach et des Méthodes Projectives. Paris, 1990. (Mimeografado)

17 - YAZIGI L \& DELPORTO JA. Bipolar disorder: The Rorschach and Karl Abraham. XIII Congrès International du Rorschach et des Méthodes Projectives. Centre de Psychologie Appliquée, Paris 1990, résumé 238.

18 - OSÓRIO LC. Identidade e identificação: Uma revisão conceitual. Rev Bras Psicanál 17:.63-81, 1983.

19 - KAPLAN HI \& SADOCK BJ. Compêndio de psiquiatria. 2a. ed., Artes Médicas. Porto Alegre, p:305-327, 1995: Disturbios de humor.

20 - FENICHEL O. Teoria psicanalítica das neuroses. Atheneu, Rio de Janeiro, p. 361-385, 1981: Depressão e mania.

Recebido para publicação em 03/10/95

Aprovado para publicação em 31/07/96 